\title{
CONTINUED COOLING OF THE CRUST IN THE NEUTRON STAR LOW-MASS X-RAY BINARY KS 1731-260
}

\author{
Edward M. Cackett ${ }^{1,2,6}$, Edward F. Brown ${ }^{3}$, Andrew Cumming ${ }^{4}$, Nathalie Degenaar $^{5}$, \\ JON M. MILLER ${ }^{1}$, AND RUDY WiJNANDS ${ }^{5}$ \\ ${ }^{1}$ Department of Astronomy, University of Michigan, 500 Church St., Ann Arbor, MI 48109-1042, USA; ecackett@ast.cam.ac.uk \\ 2 Institute of Astronomy, University of Cambridge, Madingley Rd, Cambridge CB3 OHA, UK \\ ${ }^{3}$ Department of Physics \& Astronomy, National Superconducting Cyclotron Laboratory, and the Joint Institute for Nuclear Astrophysics, \\ Michigan State University, East Lansing, MI 48824, USA \\ ${ }^{4}$ Department of Physics, McGill University, 3600 rue University, Montreal, QC, H3A 2T8, Canada \\ 5 Astronomical Institute "Anton Pannekoek," University of Amsterdam, Kruislaan 403, 1098 SJ, Amsterdam, The Netherlands \\ Received 2010 June 28; accepted 2010 September 13; published 2010 September 24
}

\begin{abstract}
Some neutron star low-mass X-ray binaries have very long outbursts (lasting several years) which can generate a significant amount of heat in the neutron star crust. After the system has returned to quiescence, the crust then thermally relaxes. This provides a rare opportunity to study the thermal properties of neutron star crusts, putting constraints on the thermal conductivity and hence the structure and composition of the crust. KS 1731-260 is one of only four systems where this crustal cooling has been observed. Here, we present a new Chandra observation of this source approximately eight years after the end of the last outburst and four years since the last observation. We find that the source has continued to cool, with the cooling curve displaying a simple power-law decay. This suggests that the crust has not fully thermally relaxed yet and may continue to cool further. A simple power-law decay is in contrast to theoretical cooling models of the crust, which predict that the crust should now have cooled to the same temperature as the neutron star core.
\end{abstract}

Key words: stars: neutron - X-rays: binaries - X-rays: individual (KS 1731-260)

\section{INTRODUCTION}

Neutron stars in transient low-mass X-ray binaries (LMXBs) provide a rare observational opportunity to study the thermal properties of the neutron star crust. In some of these transient systems accretion outbursts last many years, as opposed to the more typical weeks to months. These quasi-persistent systems are particularly interesting as during the long outbursts the crust should be heated out of thermal equilibrium with the rest of the star (Rutledge et al. 2002). Thus, once the source returns to quiescence, the crust thermally relaxes. The resulting cooling curve depends on a number of key properties of the crust including the composition and structure of the crust (Rutledge et al. 2002; Shternin et al. 2007; Brown \& Cumming 2009), and the crust thickness (which is dependent on the mass and radius of the star; Lattimer et al. 1994; Brown \& Cumming 2009).

Such crustal cooling has now been observed in four neutron star transients: KS 1731-260 (Wijnands et al. 2001, 2002; Cackett et al. 2006), MXB 1659-29 (Wijnands et al. 2003, 2004; Cackett et al. 2006, 2008), EXO 0748-676 (Degenaar et al. 2009, 2010), and XTE J1701-462 (Fridriksson et al. 2010). KS 1731-260 was in outburst for 12.5 years, returning to quiescence in 2001 (Wijnands et al. 2001). For a detailed history of this source, see Cackett et al. (2006). It was quickly realized that such a long outburst should allow for observable crustal cooling, hence, a monitoring campaign using Chandra and XMM-Newton followed (Wijnands et al. 2001, 2002; Cackett et al. 2006). A cooling curve covering the first $\sim 4$ years of quiescence was presented by Cackett et al. (2006). The source flux initially dropped rapidly: a factor of $\sim 2.5$ during the first year in quiescence and a factor of $\sim 5$ in the first 1000 days (Wijnands et al. 2002; Cackett et al. 2006). The cooling curve was well fit by either an exponential decay to a constant level

\footnotetext{
${ }^{6}$ Chandra Fellow
}

or a simple power-law decay (Cackett et al. 2006, 2008). The rapid rate of cooling and the inferred cold temperature of the neutron star core led to conclusions that the crust must have a high thermal conductivity and required enhanced levels of core cooling (Wijnands et al. 2002; Cackett et al. 2006) based on comparing with the theoretical cooling models of Rutledge et al. (2002). Motivated by these cooling curves, theoretical crust cooling models for KS 1731-260 were calculated by Shternin et al. (2007). These authors rule out a low thermal conductivity for the crust, but do not require enhanced core cooling. Brown \& Cumming (2009) also calculated thermal relaxation models for neutron star crusts, finding a high thermal conductivity for the crust and a low impurity parameter (i.e., the dispersion in the charge of the ions in the crust is low).

Here, we present a new Chandra observation of KS 1731-260, performed approximately eight years after the end of the outburst and four years since the previous Chandra observation. The data are consistent with further cooling of the neutron star, continuing along a power-law decay.

\section{ANALYSIS AND RESULTS}

The newest Chandra observation of KS 1731-260 was performed in two separate pointings. One $31 \mathrm{ks}$ segment was performed on 2009 May 17 (ObsID: 10037) and the other $28 \mathrm{ks}$ segment was performed on 2009 May 19 (ObsID: 10911). The source was at the default aim point on the ACIS-S3 chip, which was operated in FAINT mode. Given several recent changes to the calibration of Chandra, we have opted to also reanalyze all the previous Chandra observations with the latest calibration and software. Moreover, we reanalyze the $X M M$-Newton observations with the latest calibration files and software. For full details of the previous observations, please refer to Cackett et al. (2006). 
Table 1

Neutron Star Atmosphere Spectral Fitting Parameters

\begin{tabular}{|c|c|c|c|c|c|c|c|c|}
\hline Parameter & $\begin{array}{c}2428 \\
(C X O)\end{array}$ & $\begin{array}{c}013795201 / 301 \\
(X M M)\end{array}$ & $\begin{array}{c}3796 \\
(C X O)\end{array}$ & $\begin{array}{c}3797 \\
(C X O)\end{array}$ & $\begin{array}{c}0202680101 \\
(X M M)\end{array}$ & $\begin{array}{c}6279 \\
(\mathrm{CXO})\end{array}$ & $\begin{array}{c}5468 \\
(C X O)\end{array}$ & $\begin{array}{c}10037 / 10911 \\
(C X O)\end{array}$ \\
\hline$\overline{M J D}$ & 51995.1 & 52165.7 & 52681.6 & 52859.5 & 53430.5 & 53500.4 & 53525.4 & 54969.7 \\
\hline$N_{\mathrm{H}}\left(10^{22} \mathrm{~cm}^{-2}\right)$ & \multicolumn{8}{|c|}{$1.30 \pm 0.06$} \\
\hline$k T_{\mathrm{eff}}^{\infty}(\mathrm{eV})$ & $103.2 \pm 1.7$ & $88.9 \pm 1.3$ & $75.5 \pm 2.2$ & $73.3 \pm 2.3$ & $71.0 \pm 1.8$ & $66.0 \pm 4.5$ & $70.3 \pm 2.1$ & $63.1 \pm 2.1$ \\
\hline$F_{\text {bol }^{\text {a }}}\left(10^{-13} \mathrm{erg} \mathrm{cm}^{-2} \mathrm{~s}^{-1}\right)$ & $4.2 \pm 0.3$ & $2.3 \pm 0.1$ & $1.2 \pm 0.1$ & $1.1 \pm 0.1$ & $0.95 \pm 0.09$ & $0.72 \pm 0.21$ & $0.92 \pm 0.11$ & $0.60 \pm 0.08$ \\
\hline
\end{tabular}

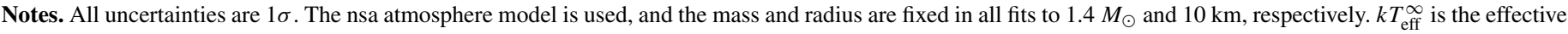

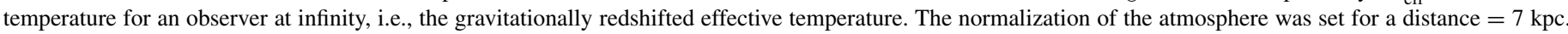
ObsIDs of each observation are indicated at the top, with CXO (Chandra) and XMM (XMM-Newton) denoting the observatory.

a Bolometric flux calculated from the model over the $0.01-100 \mathrm{keV}$ range.

\subsection{Chandra Data Reduction}

The Chandra data were all analyzed using CIAO (v 4.2) and CALDB (v 4.2.2). We used a circular source extraction region with a radius of $3^{\prime \prime}$, and the background extraction region was an annulus with an inner radius of 7 " and an outer radius of $25^{\prime \prime}$. The psextract tool was used to extract the spectra, and mkacisrmf and mkarf were used to create the response matrices.

\subsection{XMM-Newton Data Reduction}

The XMM-Newton data were analyzed using the XMM Science Analysis Software (v 9.0.0). The observation data files were reprocessed using the emproc and epproc tasks. To check for high levels of background flaring, we extracted light curves for all events with $>10 \mathrm{keV}$ and pattern $=0$ for the MOS, and $10-12 \mathrm{keV}$ and pattern $=0$ for the PN. In all observations there was some significant background flaring. We excluded all times where the $>10 \mathrm{keV}$ light curve was greater than 2 counts $\mathrm{s}^{-1}$ for the MOS and 4 counts $\mathrm{s}^{-1}$ for the PN. We extracted spectra using evselect with a circular source extraction region of radius $10^{\prime \prime}$ and a circular background extraction region of radius $1^{\prime}$ taken from a source free region close to the source. For the MOS, we filtered with patterns 0-12, and for the PN, we used patterns $0-4$ and flag $=0$. Response matrices were generated using rmfgen and arfgen.

\subsection{Spectral Analysis}

We fit the spectra using XSPEC (ver. 12; Arnaud 1996), following similar procedures to Cackett et al. (2006) and Cackett et al. (2008). The spectra were modeled with an absorbed neutron star atmosphere model. We used the phabs model for Galactic absorption and the nsa model for the neutron star atmosphere (Zavlin et al. 1996). Throughout, we fix the neutron star radius at $10 \mathrm{~km}$ and the neutron star mass at $1.4 M_{\odot}$. The normalization of the nsa model is given by $1 / D^{2}$, where $D$ is the distance to the source in pc. Here, we assume a distance of $7 \mathrm{kpc}$ (Muno et al. 2000), i.e., normalization $=2.041 \times$ $10^{-8} \mathrm{pc}^{-2}$. As we showed for MXB 1659-29 in Cackett et al. (2008), the distance assumed only shifts the fitted temperatures up or down and does not affect the cooling timescales derived. Here, if we assume a distance of $5 \mathrm{kpc}$, the temperatures are all about $10 \%$ lower than for $D=7 \mathrm{kpc}$, where as if we assume $D=9 \mathrm{kpc}$ then the temperatures are approximately $7 \%$ higher than for $D=7 \mathrm{kpc}$.

We fit all the spectra simultaneously with the absorption column density, $N_{\mathrm{H}}$, the same for all spectra and a free parameter in the fit. The neutron star atmosphere effective temperature is allowed to vary between epochs. For the first XMM-Newton observations (013795201/013795301), the two sets of spectra had their parameters tied between them. Similarly, for the last Chandra observation $(10037 / 10911)$ we also tie the parameters between the two spectra. Given the low count rates, there are not enough counts per bin to use $\chi^{2}$ statistics when fitting, and therefore we use the $W$-statistic in XSPEC to fit the unbinned spectra.

The results of the spectral fitting are given in Table 1, and the evolution of the effective temperature is shown in Figure 1. All uncertainties quoted and plotted are at the $1 \sigma$ level of confidence. Note that in Cackett et al. (2006) the uncertainties quoted were at the $90 \%$ level of confidence, not $1 \sigma$ as stated in the text. The results clearly show a decrease in the neutron star atmosphere temperature over time, with the newest observation the coldest yet.

\subsection{Count Rate Analysis}

One potential problem with our interpretation of crustal cooling is that there could be a power-law spectral component that cannot be detected here because of the low number of counts in the spectra. Non-thermal power-law components are common in many quiescent neutron star spectra (e.g., Jonker et al. 2004). Any power-law component could potentially change the shape of the cooling curve of the thermal component. It should be noted that the highest quality spectra we have of KS 1731-260 is the first Chandra observation. This observation was discussed in detail by Wijnands et al. (2001). These authors show that the spectrum is consistent with a thermal spectrum only, and a power law is not required statistically. However, if they add a power law to their model they find that it only contributes $\sim 15 \%$ to the flux. Due to the low count rate of the later observations, here, we use count ratios between different bands to assess whether there could be a significant power-law component present. One can only do this with observations from the same telescope (Chandra and XMM-Newton have different effective areas), thus we analyze just the raw counts from the six Chandra observations.

Figure 2 shows Chandra count rate versus time. This light curve also shows a power-law decay like the temperatures from spectral fitting. We find that only the very first observation has a significant detection in the $3-10 \mathrm{keV}$ band where the power-law component would dominate. To study the spectral evolution of the source, we therefore define a hardness color ratio between the count rate in the $1.5-3.0 \mathrm{keV}$ band and the $0.5-1.5 \mathrm{keV}$ band. In Figure 3, we show a hardness-intensity diagram that displays the evolution of this color with the full $0.5-10 \mathrm{keV}$ count rate. We also show the predicted color and count rate evolution for three different spectral models: (1) a neutron star atmosphere, (2) a neutron star atmosphere plus power-law model where the power law always contributes $20 \%$ to the unabsorbed $0.5-10 \mathrm{keV}$ flux 


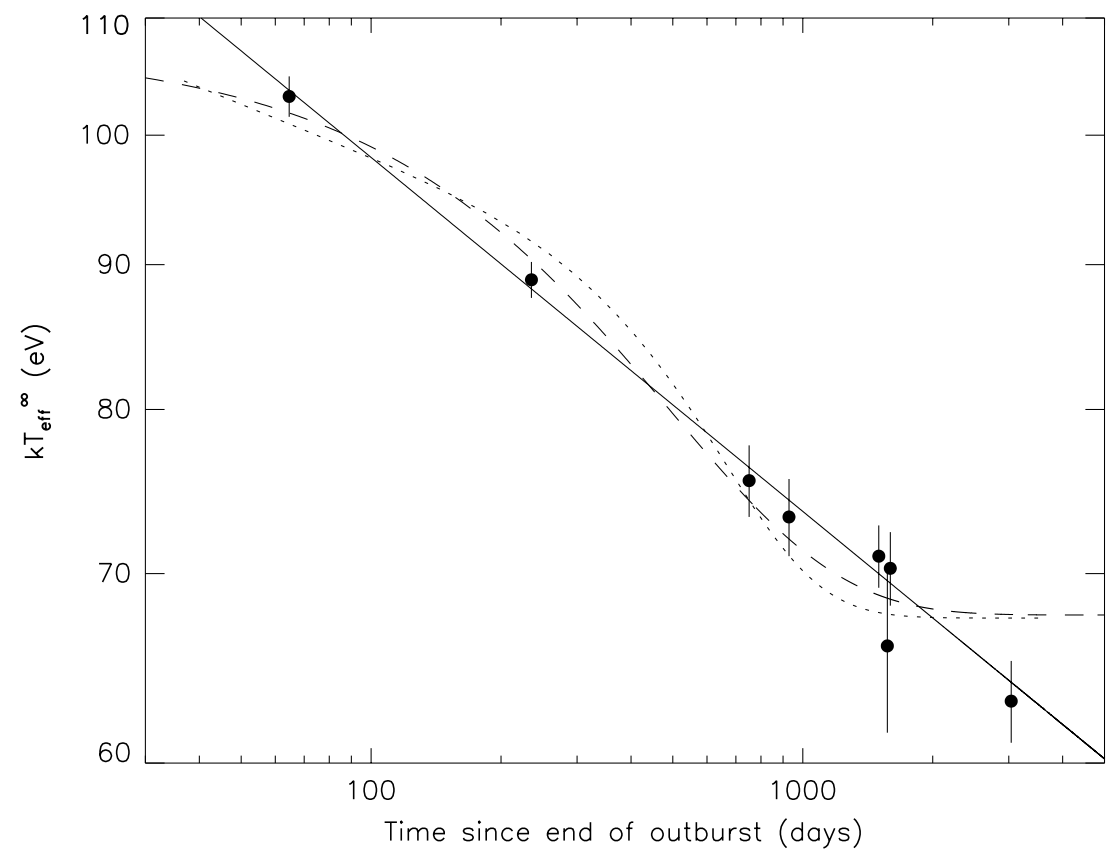

Figure 1. Effective temperature for an observer at infinity for KS 1731-260 over approximately 3000 days from the end of the last outburst. The solid line shows a power-law fit to these temperatures, while the dashed line shows the best-fitting exponential decay to a constant level. The dotted line shows the best-fitting model using the crustal cooling simulations from Brown \& Cumming (2009).

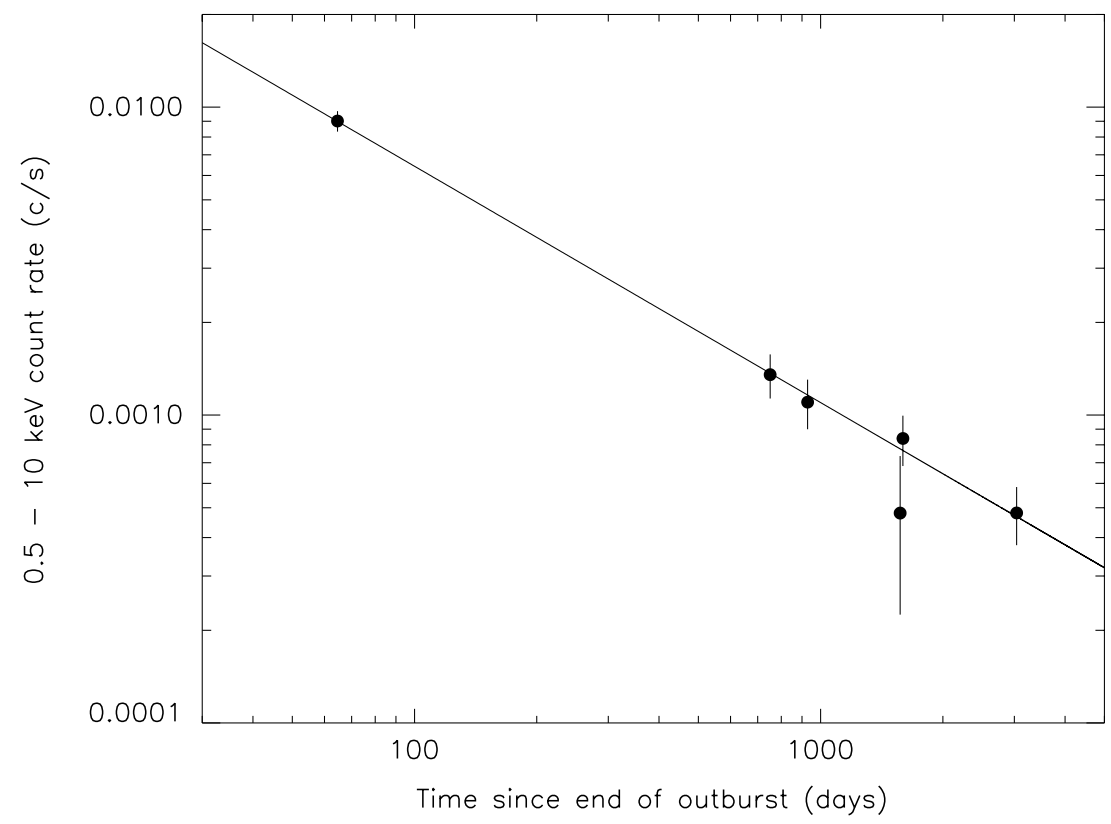

Figure 2. $0.5-10 \mathrm{keV}$ count rates for all Chandra observations of KS 1731-260. The solid line shows a power-law fit to these count rates.

(we assume a spectral index, $\Gamma=2$ ), and (3) a neutron star atmosphere plus power-law $(\Gamma=2)$ model where the powerlaw flux always contributes $40 \%$ of the unabsorbed $0.5-10 \mathrm{keV}$ flux (note that all three models assume Galactic absorption of $N_{\mathrm{H}}=1.3 \times 10^{22} \mathrm{~cm}^{-2}$ ). The data points are most consistent with a simple cooling neutron star atmosphere, but model (2), where there is a small contribution from a power law, cannot be ruled out. Any power-law contribution increases the color (for a given count rate) compared to the color from a neutron star atmosphere only. A harder power-law spectrum (with a slope closer to 1 than 2) would also lead to an increased color. Thus, any contributions from a power-law component must be at a low level (less than a few tens of percent). Therefore, there must still be significant neutron star cooling. Also note that this count rate analysis does not take into account any change in the effective area of the detector over time. There has been a known increase in contaminant on the Chandra ACIS detector since the mission launch, leading to a decrease in the sensitivity at lowest energies over time (Marshall et al. 2004). This would artificially harden the color used here, thus the colors shown here should be taken as maximum values.

To further test the affect of a constant fraction of power-law flux, we fit the spectra with an absorbed neutron star atmosphere plus power-law model. We fix the power law with slope $\Gamma=2$, and the normalization for each observation is set to give a $0.5-10 \mathrm{keV}$ unabsorbed flux that is $20 \%$ of the value found 


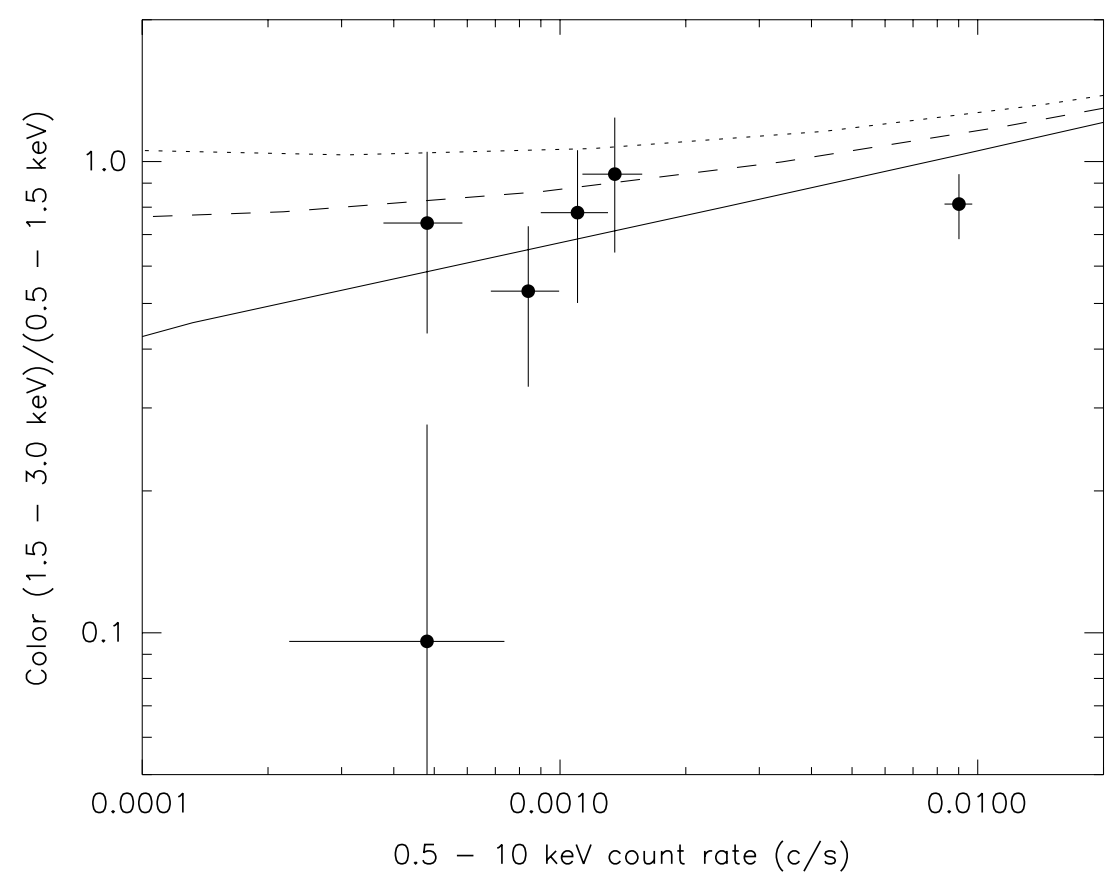

Figure 3. Hardness-intensity diagram for Chandra observations of KS 1731-260. The hardness color is the ratio of the $1.5-3.0 \mathrm{keV}$ count rate to the $0.5-1.5 \mathrm{keV}$ count rate. This is plotted against the count rate for the $0.5-10 \mathrm{keV}$ band. The solid line shows the predicted count rates for a cooling neutron star atmosphere. The dashed line shows the predicted count rates for a neutron star atmosphere and power-law spectrum, where the power law $(\Gamma=2)$ always contributes $20 \%$ to the unabsorbed $0.5-10 \mathrm{keV}$ flux. The dotted line shows a neutron star atmosphere plus power-law spectrum $(\Gamma=2)$ where the power-law flux always contributes $40 \%$ to the unabsorbed $0.5-10 \mathrm{keV}$ flux.

from fitting a neutron star atmosphere alone. The resulting temperatures still follow a simple power-law decay cooling curve, with the same slope as before. This can be easily understood, as reducing the thermal flux by $20 \%$ will simply just reduce the measured temperature by approximately $5 \%$ as $F \propto T^{4}$.

\subsection{Cooling Curves}

The latest Chandra observation analyzed here was taken approximately four years after the nearest observations and appears to show continued cooling of the neutron star. We compare the three observations taken around MJD 53,500 with this newest observation. These three observations (the fifth, sixth, and seventh quiescent observations) were all taken within 100 days of each other, and all have consistent temperatures at the $1 \sigma$ level. A weighted average of the temperatures from these three observations gives $k T_{\text {eff }}^{\infty}=70.3 \pm 1.3 \mathrm{eV}$. Compared to the latest Chandra observation, where $k T_{\text {eff }}^{\infty}=63.1 \pm 2.1$ $\mathrm{eV}$, the two temperatures differ at the $3 \sigma$ level of confidence, suggesting continued cooling of KS 1731-260.

Before this latest observation, the cooling curve could be well fit by either a power law (Cackett et al. 2008) or an exponential decay to a constant level (Cackett et al. 2006). We tested both forms here. We fitted a power law of the form $y(t)=\alpha\left(t-t_{0}\right)^{\beta}$ to the effective temperatures, ${ }^{7}$ where we chose $t_{0}$ to be midday on the last day the source was observed to be active (MJD 51,930.5; see Cackett et al. 2006). The best-fitting parameters are $\alpha=174.7 \pm 1.3 \mathrm{eV}$ and $\beta=-0.125 \pm 0.007$, giving $\chi_{v}^{2}=0.33, P_{\chi}=0.92$. This best-fitting power law is shown in Figure 1 as a solid line and fits the cooling curve well. For comparison, we also test an exponential decay of the form $y(t)=a \exp \left[-\left(t-t_{0}\right) / b\right]+c$. The best-fitting parameters are

\footnotetext{
7 Note that as $F \propto T^{4}$ the flux decay curve can simply be calculated from the fit to the temperatures.
}

$a=39.8 \pm 2.3 \mathrm{eV}, b=418 \pm 70$ days, and $c=67.7 \pm$ $1.3 \mathrm{eV}$, giving $\chi_{v}^{2}=2.0, P_{\chi}=0.04$. The simple powerlaw decay is therefore a better fit to the cooling curve, further indicating continued cooling of KS 1731-260.

We also fitted numerical crust cooling simulations from Brown \& Cumming (2009) to the data. These calculations by Brown \& Cumming (2009) suggest that the light curve of a cooling crust is expected to be a broken power law which flattens to a constant at late times, set by the temperature of the neutron star core. The initial power-law decay is set by the temperature profile in the outer crust. The power-law break occurs when there is a transition in the solid from a classical to quantum crystal at a depth close to neutron drip. The time of this power-law break is set by the thermal diffusion time to the depth of this transition. In these models the three variable parameters are (1) the crust impurity parameter which is given by $Q_{\text {imp }} \equiv n_{\text {ion }}^{-1} \sum_{i} n_{i}\left(Z_{i}-\langle Z\rangle\right)^{2}$ and measures the dispersion in the charge of the nuclides $(Z)$ in the crust, (2) the core temperature, $T_{c}$, and (3) the temperature at the top of the crust, $T_{b}$. The best-fitting model (dotted line in Figure 1) has $Q_{\text {imp }}=4.0, T_{c}=5.4 \times 10^{7} \mathrm{~K}$, and $T_{b}=2 \times 10^{8} \mathrm{~K}$ (at a column of $1 \times 10^{12} \mathrm{~g} \mathrm{~cm}^{-2}$ during the outburst). This model gives $\chi_{v}^{2}=3.3$. These values are quite similar to the best fit in Brown $\&$ Cumming (2009) who found $Q_{\text {imp }}=1.5, T_{c}=4.6 \times 10^{7} \mathrm{~K}$, and $T_{b}=2.5 \times 10^{8} \mathrm{~K}$. Thus, the core temperature here is slightly colder and the impurity parameter in the crust is slightly higher than the previous best-fitting values.

\section{DISCUSSION AND CONCLUSIONS}

We have presented a new Chandra observation of the neutron star LMXB KS 1731-260 in quiescence. This observation extends the monitoring of this source to approximately eight years after the end of the most recent outburst. The observation suggests that the neutron star has continued to cool with an 
effective temperature (for an observer at infinity) of $k T_{\text {eff }}^{\infty}=$ $63.1 \pm 2.1 \mathrm{eV}$ compared to the preceding epoch where $k T_{\text {eff }}^{\infty}=$ $70.3 \pm 1.3 \mathrm{eV}$. Previously, the cooling curve could be well fit by both a power-law decay or an exponential decay to a constant level (Cackett et al. 2006, 2008). Here, we find that the latest observation remains on the simple power-law decay with a slope of $-0.125 \pm 0.007$, indicating that the crust may be continuing to cool and has not yet reached thermal equilibrium with the core. As has been discussed previously (Wijnands et al. 2002; Cackett et al. 2006; Shternin et al. 2007; Brown \& Cumming 2009), the cooling of KS 1731-260 indicates a crust with high thermal conductivity. This implies that the crust has a low impurity parameter, in other words, the dispersion in the charge of the ions in the crust is low (Brown \& Cumming 2009, find $Q_{\text {imp }}<10$ is a robust upper limit).

One potential problem is that the spectrum may not be purely thermal, but that there could also be some contribution from nonthermal power-law emission that cannot be detected due to low number of counts in the spectra. Recent observations of another cooling neutron star XTE J1701-462 have shown an increase in flux for approximately 100 days on top of a cooling curve, likely due to increased levels of residual accretion (Fridriksson et al. 2010). Furthermore, long-term monitoring of the neutron star transient Cen X-4 in quiescence has shown variability in the thermal component that cannot be explained by crustal cooling and may also be linked to residual accretion during quiescence (Cackett et al. 2010). The monitoring of KS 1731-260 is relatively sparse (only eight observations over eight years) and thus any increase in flux similar to XTE J1701-462 could easily have been missed. However, only the first observation of KS 1731-260 shows a significant detection above $3 \mathrm{keV}$, and in this observation Wijnands et al. (2001) found that the contribution of the power law was limited to about $15 \%$ of the flux, though the power-law component was not statistically required to fit the spectrum. In order to investigate the potential contribution from power-law emission, we created a hardness-intensity diagram using only the Chandra count rates in different bands. The data are consistent with a cooling neutron star atmosphere, though a small contribution (a few tens of percent) from a powerlaw component cannot be excluded.

From a theoretical perspective, it is puzzling that the decay is best fit by a single power law. Numerical simulations of crustal cooling expect that the light curve should approximate a broken power law which flattens to a constant at late times (Brown \& Cumming 2009). The break in the power law is caused by a change from classical to quantum crystals, and the timescale on which this occurs is set by the thermal diffusion time to the depth of this transition. Though in KS 1731-260 we only see a single, unbroken power-law decay in the light curve, fitting the model of Brown \& Cumming (2009) allows for quantitative constraints on the thermal conductivity of the crust. The best-fitting model gives an impurity parameter $Q_{\text {imp }}=4.0$ and core temperature $T_{c}=5.4 \times 10^{7} \mathrm{~K}$ (note that these values are for a fixed mass and radius, and any increase in the surface gravity shortens the cooling time by decreasing the crust thickness). The impurity parameter is marginally higher and the core temperature is slightly lower than the best-fitting values in Brown \& Cumming (2009). The additional Chandra observation requires a lower core temperature to fit the light curve at late times, where as the impurity parameter needs to be increased to maintain the thermal cooling timescale (a lower core temperature increases the thermal conductivity and thus increasing the impurity parameter compensates). The implied core temperature only requires standard models for core cooling without enhanced levels on neutrino cooling (Shternin et al. 2007; Brown \& Cumming 2009).

It is interesting to note that a power-law decay with a slope of $1 / 8$ (as observed here) is expected for thermal relaxation of the core by enhanced neutrino emission (i.e., the rate goes as $T^{6}$, see, e.g., Page et al. 2006). Such a scenario requires the core to be heated up substantially during an outburst. However, in order to do this, or to cool the core over the observed timescale, would require a core specific heat several orders of magnitude smaller than that provided by degenerate electrons, assuming an electron fraction of order 0.1 .

E.M.C. thanks the staff at Durham University for kind hospitality during a visit where much of this work was completed. E.M.C. gratefully acknowledges support provided by NASA through the Chandra Fellowship Program.

\section{REFERENCES}

Arnaud, K. A. 1996, in ASP Conf. Ser. 101, Astronomical Data Analysis Software and Systems V, ed. G. H. Jacoby \& J. Barnes (San Francisco, CA: ASP), 17

Brown, E. F., \& Cumming, A. 2009, ApJ, 698, 1020

Cackett, E. M., Brown, E. F., Miller, J. M., \& Wijnands, R. 2010, ApJ, 720 , 1325

Cackett, E. M., Wijnands, R., Linares, M., Miller, J. M., Homan, J., \& Lewin, W. H. G. 2006, MNRAS, 372, 479

Cackett, E. M., Wijnands, R., Miller, J. M., Brown, E. F., \& Degenaar, N. 2008, ApJ, 687, L87

Degenaar, N., et al. 2009, MNRAS, 396, L26

Degenaar, N., et al. 2010, MNRAS, in press (arXiv:1007.0247)

Fridriksson, J. K., et al. 2010, ApJ, 714, 270

Jonker, P. G., Galloway, D. K., McClintock, J. E., Buxton, M., Garcia, M., \& Murray, S. 2004, MNRAS, 354, 666

Lattimer, J. M., van Riper, K. A., Prakash, M., \& Prakash, M. 1994, ApJ, 425, 802

Marshall, H. L., Tennant, A., Grant, C. E., Hitchcock, A. P., O’Dell, S. L., \& Plucinsky, P. P. 2004, Proc. SPIE, 5165, 497

Muno, M. P., Fox, D. W., Morgan, E. H., \& Bildsten, L. 2000, ApJ, 542, 1016

Page, D., Geppert, U., \& Weber, F. 2006, Nucl. Phys. A, 777, 497

Rutledge, R. E., Bildsten, L., Brown, E. F., Pavlov, G. G., Zavlin, V. E., \& Ushomirsky, G. 2002, ApJ, 580, 413

Shternin, P. S., Yakovlev, D. G., Haensel, P., \& Potekhin, A. Y. 2007, MNRAS, 382, L43

Wijnands, R., Guainazzi, M., van der Klis, M., \& Méndez, M. 2002, ApJ, 573, L45

Wijnands, R., Homan, J., Miller, J. M., \& Lewin, W. H. G. 2004, ApJ, 606, L61

Wijnands, R., Miller, J. M., Markwardt, C., Lewin, W. H. G., \& van der Klis, M. 2001, ApJ, 560, L159

Wijnands, R., Nowak, M., Miller, J. M., Homan, J., Wachter, S., \& Lewin, W. H. G. 2003, ApJ, 594, 952

Zavlin, V. E., Pavlov, G. G., \& Shibanov, Y. A. 1996, A\&A, 315, 141 